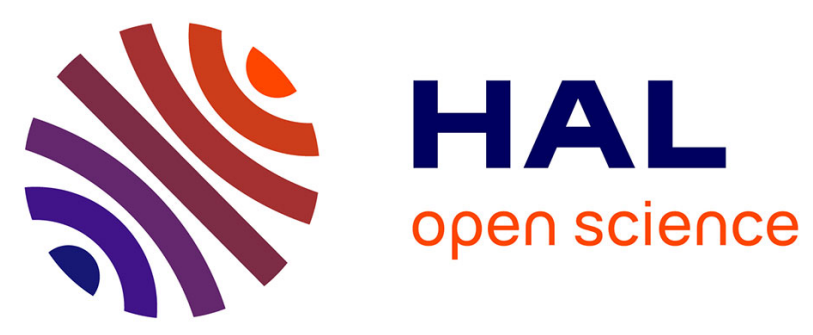

\title{
Robustness Analysis of the Collective Dynamics of Nonlinear Periodic Structures Under Parametric Uncertainty
}

Khaoula Chikhaoui, Diala Bitar, Najib Kacem, Noureddine Bouhaddi

\section{To cite this version:}

Khaoula Chikhaoui, Diala Bitar, Najib Kacem, Noureddine Bouhaddi. Robustness Analysis of the Collective Dynamics of Nonlinear Periodic Structures Under Parametric Uncertainty. ASME 2016 International Mechanical Engineering Congress and Exposition, Nov 2016, Phoenix, United States. 10.1115/IMECE2016-66720 . hal-01512528

\section{HAL Id: hal-01512528 \\ https://hal.science/hal-01512528}

Submitted on 23 Apr 2017

HAL is a multi-disciplinary open access archive for the deposit and dissemination of scientific research documents, whether they are published or not. The documents may come from teaching and research institutions in France or abroad, or from public or private research centers.
L'archive ouverte pluridisciplinaire HAL, est destinée au dépôt et à la diffusion de documents scientifiques de niveau recherche, publiés ou non, émanant des établissements d'enseignement et de recherche français ou étrangers, des laboratoires publics ou privés. 


\section{ROBUSTNESS ANALYSIS OF THE COLLECTIVE DYNAMICS OF NONLINEAR PERIODIC STRUCTURES UNDER PARAMETRIC UNCERTAINTY}

\author{
Khaoula Chikhaoui \\ FEMTO-ST Institute, UMR 6174, Department of \\ Applied Mechanics, Univ. Bourgogne Franche- \\ Comté, 24 Rue de l'Epitaphe 25000 Besançon, \\ France \\ khaoula.chikhaoui@femto-st.fr
}

\section{Najib Kacem}

FEMTO-ST Institute, UMR 6174, Department of Applied Mechanics, Univ. Bourgogne FrancheComté, 24 Rue de l'Epitaphe 25000 Besançon,

$$
\text { France }
$$

najib.kacem@femto-st.fr

\author{
Diala Bitar \\ FEMTO-ST Institute, UMR 6174, Department of \\ Applied Mechanics, Univ. Bourgogne Franche- \\ Comté, 24 Rue de l'Epitaphe 25000 Besançon, \\ France \\ diala.bitar@femto-st.fr
}

\section{Noureddine Bouhaddi}

FEMTO-ST Institute, UMR 6174, Department of Applied Mechanics, Univ. Bourgogne FrancheComté, 24 Rue de l'Epitaphe 25000 Besançon, France noureddine.bouhaddi@femto-st.fr

\section{ABSTRACT}

In order to ensure more realistic design of nonlinear periodic structures, the collective dynamics of a coupled pendulums system is investigated under parametric uncertainties. A generic discrete analytical model combining the multiple scales method, the perturbation theory and a standing-wave decomposition is proposed and adapted to the presence of uncertainties. These uncertainties are taken into account through a probabilistic modeling implying that the stochastic parameters vary according to random variables of chosen probability density functions. The proposed model leads to a set of coupled complex algebraic equations written according to the number and positions of the uncertainties in the structure and numerically solved using the time integration Runge-Kutta method. The uncertainty propagation through the established model is finally ensured using the Latin Hypercube Sampling method. The analysis of the dispersion, in term of variability of the frequency and amplitude intervals of the multistability domain shows the effects of uncertainties on the stability and nonlinearity of a three coupled pendulums structure. The nonlinear aspect is strengthened, the multistability domain is wider, more stable branches are obtained and thus the multimode solutions are enhanced.
Keywords: uncertainty, nonlinearity, periodic structure, collective dynamics

\section{INTRODUCTION}

Under the hypothesis of perfect periodicity, many engineering researches provided interesting insights in the dynamic analysis of nonlinear periodic structures. For instance, a straight-forward perturbation analysis was applied by Boechler et al. [1] to investigate amplitude-dependent dispersion of a discrete one dimensional nonlinear periodic chain with Hertzian contact. Moreover, the multiple scales approach was combined with the secular perturbation theory, in a discrete model, by Lifshitz and Cross [2] to calculate the responses of a coupled array of nonlinear oscillators under parametric excitation and recently by Bitar et al. [3] to investigate the collective dynamics of a periodic structure of coupled nonlinear Duffing-Van Der Pol oscillators under simultaneous parametric and external excitations. Multiple scales perturbation approach was also used by Gutschmidt and Gottlied [4] in a continuum-based model to investigate the dynamic behavior of an array of $\mathrm{N}$ nonlinearly coupled microbeams and by Manktelow et al. [5] to study the wave interactions in diatomic chain with two degrees of freedom per unit cell. Recently, Andreassen et al. [6] studied the wave interactions in a periodically perforated plate through the two- 
dimensional dispersion characteristics, group velocities and internal resonances investigation.

Nevertheless, far from the mathematical idealization due to the perfect periodicity hypothesis, imperfections, which can be due to material defects, structural damage, ageing, fatigue, manufacturing processes, etc., and which reflect the nature of real engineering systems, can perturb the perfect arrangement of cells in a structure and change significantly the dynamic behavior from the predictions done under perfect periodicity. In the literature, Kissel [7], for instance, investigated the effects of the disorder in one-dimensional periodic structure theoretically and numerically using Monte Carlo (MC) simulations and proved that the disorder causes wave attenuation and pronounced spatial localization of normal modes at frequencies near the stopbands of the perfectly periodic associated structure. Statistical investigation of the effect of disorder on the dynamics of multidimensional coupled periodic structures, using the MC method, was carried out by Cha et al. [8]. Moreover, to study the effects of the randomness of flexible joints on the free vibrations of simply-supported periodic large space trusses, Koch [9] combined an extended Timoshenko beam continuum model, $\mathrm{MC}$ simulations and first-order perturbation method.

Nonlinear coupled pendulums arrays are widely used in mechanical engineering and have been purpose of several researches in the literature. For instance, Jallouli et al. [10] investigated the nonlinear dynamics of a two-dimensional array of coupled pendulums under parametric excitation. Moreover, in the context of imperfections, Tjavaras and Triantafyllou [11] investigated numerically the effect of nonlinearities on the forced response of two disordered pendula coupled through a weak linear spring. Hai-Quing and Yi [12] developed a discrete theoretical model based on the envelope function approach to study analytically and numerically the effect of mass impurity on nonlinear localized modes in a chain of parametrically driven coupled pendula. The influence of impurities on the envelope waves in a driven nonlinear pendulum chain has been investigated numerically under a continuum-limit approximation in [13] and experimentally in [14].

Extending the framework to the dispersion analysis of nonlinear periodic structures dynamics under parametric uncertainty is a complex challenge and the main aim of this work is to deal with. Therefore, based on the work performed by Diala et al. [15] and under the hypothesis of small imperfections which implies that the collective dynamics and the localization phenomena due to the weak coupling of components are not destroyed, the present paper is focused on the collective dynamics analysis of a stochastic periodic nonlinear structure composed of coupled pendulums. A generic discrete analytical model combining the multiple scales method, the perturbation theory and a standing-wave decomposition is proposed and adapted to the presence of uncertainties. The latter are taken into account, in a probabilistic framework, as parametric uncertainties modeled by random variables. The obtained analytical model leads to a set of coupled complex algebraic equations written according to the number and positions of the uncertainties in the structure and then numerically solved using the time integration RungeKutta method. To propagate uncertainties through the established model, the statistical Latin Hypercube Sampling method [16] is used. To illustrate the stochastic effect on the dynamic behavior of a three coupled pendulums structure, when the length of the first pendulum is supposed to be uncertain, a statistical analysis of the frequency responses dispersions, both in modal and physical coordinates, is carried out.

\section{MODEL}

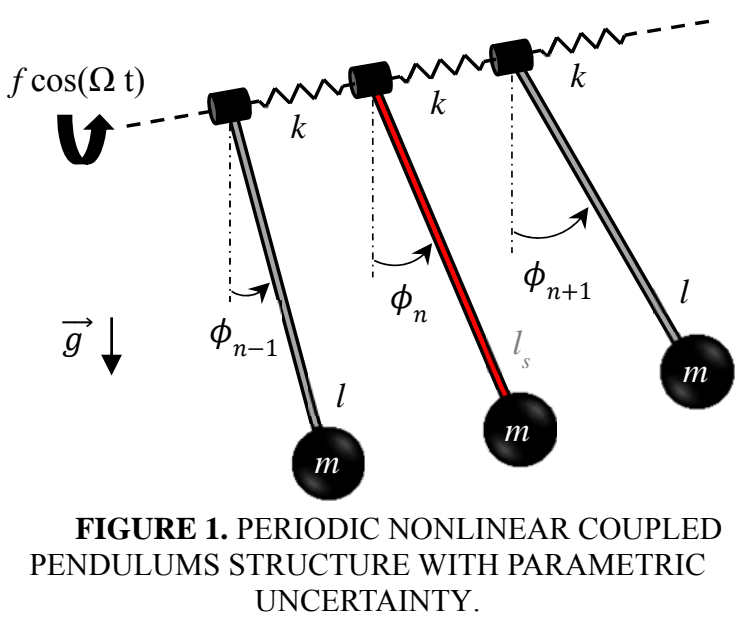

Figure 1 illustrates a generic structure of $N$ coupled pendulums of identical mass $m$ and viscous damping coefficient $c$ generated by the dissipative force acting on the supporting point of each one. The pendulums are coupled by linear springs of stiffness $k$ and are subject to an external excitation $f \cos (\Omega t)$ each one. The inclination angle $\phi_{n}$ from the equilibrium position quantifies the rotational displacement of the $n^{\text {th }}$ pendulum. Applied boundary conditions are such as the pendulums labeled 0 and $N+1$ are fixed so that $\phi_{0}=$ $\phi_{N+1}=0$. The periodicity of the structure is broken by the presence of $p$ pendulums containing parametric uncertainties, which can, for instance, be the pendulum length $l_{s}$ as illustrated in Fig. 1.

\section{Equation of motion}

Applying the Lagrange approach leads to the equation of motion of the $n^{\text {th }}$ pendulum of the form

$$
\ddot{\phi}_{n}+c_{n} \dot{\phi}_{n}+\omega_{n}^{2} \phi_{n}+K_{n} \mathcal{L}_{c}(\phi, \tilde{\phi})+\alpha_{n} \phi_{n}{ }^{3}=F_{n} \cos (\Omega t)(1)
$$

where $c_{n}=\frac{\alpha}{m l}, K_{n}=\frac{k}{m l^{2}}, \alpha_{n}=-\frac{g}{6 l}, F_{n}=\frac{f}{m l^{2}}$ when the $n^{t h}$ pendulum is deterministic and $c_{n}=\frac{\alpha}{m l_{s}}, K_{n}=\frac{k}{m l_{s}^{2}}, \alpha_{n}=-\frac{g}{6 l_{s}}$, 
$F_{n}=\frac{f}{m l_{s}{ }^{2}}$ when, for instance, the length of the $n^{t h}$ pendulum, of stochastic displacement $\tilde{\phi}_{n}$, is uncertain.

Since the linear coupling between the pendulums is supposed to be very weak and under the hypothesis of small imperfections, each frequency $\omega_{n}$ is supposed to be equal to the eigenfrequency $\omega_{0}\left(\omega_{n}=\omega_{0}=\sqrt{\frac{g}{l}}\right)$.

The linear coupling term $\mathcal{L}_{c}(\phi, \tilde{\phi})$ depends on the position of the stochastic pendulums in the chain. In fact, considering the case where the stochastic pendulums are not adjacent, four different configurations are distinguished:

a. If the concerned pendulum is deterministic as well as its neighbors, $\mathcal{L}_{c}(\phi, \tilde{\phi})=2 \phi_{n}-\phi_{n-1}-\phi_{n+1}$;

b. If the concerned pendulum is deterministic but the previous one is stochastic, $\mathcal{L}_{c}(\phi, \tilde{\phi})=2 \phi_{n}-\tilde{\phi}_{n-1}-\phi_{n+1}$;

c. If the concerned pendulum is deterministic but the following is stochastic, $\mathcal{L}_{c}(\phi, \tilde{\phi})=2 \phi_{n}-\phi_{n-1}-\tilde{\phi}_{n+1}$;

d. If the stochastic pendulum is concerned, the deterministic displacement $\phi_{n}$ is replaced by the stochastic one, $\tilde{\phi}_{n}$, in Eq. (1) such as

$$
\begin{gathered}
\ddot{\tilde{\phi}}_{n}+c_{n} \dot{\tilde{\phi}}_{n}+\omega_{0}^{2} \tilde{\phi}_{n}+K_{n} \mathcal{L}_{c}(\phi, \tilde{\phi})+\alpha_{n} \tilde{\phi}_{n}{ }^{3}=F_{n} \cos (\Omega t) \\
\text { and } \mathcal{L}_{c}(\phi, \tilde{\phi})=2 \tilde{\phi}_{n}-\phi_{n-1}-\phi_{n+1}, \text { in this case }
\end{gathered}
$$

\section{Numerical Solving}

The multiple scales method consists on replacing the single time variable by an infinite sequence of independent time scales $\left(T_{i}=\boldsymbol{\varepsilon}^{i} t\right)$, where $\boldsymbol{\varepsilon}$ is a dimensionless parameter assumed to be small, and eliminating secular terms in the fast time variable $t$.

The scaled equation of motion of the $n^{\text {th }}$ pendulum, with respect to each configuration mentioned above, is assumed as follows

$$
\begin{gathered}
\ddot{\phi}_{n}+\varepsilon c_{n} \dot{\phi}_{n}+\omega_{0}^{2} \phi_{n}+\varepsilon K_{n} \mathcal{L}_{c}(\phi, \tilde{\phi})+\varepsilon \alpha_{n} \phi_{n}{ }^{3} \\
=\varepsilon F_{n} \cos (\Omega t)
\end{gathered}
$$

where the excitation frequency $\Omega$ is expressed as $\Omega=\omega_{0}+\varepsilon \sigma$ with $\sigma$ being the detuning parameter.

The solution of Eq. (3) can generally be given by a formal power series expansion $\phi_{n}=\sum_{i} \varepsilon^{i} \phi_{i_{n}}$. Up to the first order, one obtains an equation of the form

$$
\begin{gathered}
\frac{\partial^{2} \phi_{1_{n}}}{\partial t^{2}}+\omega_{0}^{2} \phi_{1_{n}}+c_{n} \frac{\partial \phi_{0_{n}}}{\partial t}+2 \frac{\partial^{2} \phi_{0_{n}}}{\partial t \partial T}+K_{n} \mathcal{L}_{c}\left(\phi_{0}, \tilde{\phi}_{0}\right) \\
+\alpha_{n} \phi_{0_{n}}^{3}=\frac{F_{n}}{2} \exp \left[i\left(\omega_{0} t+\sigma T\right)\right]
\end{gathered}
$$

For simplicity, Eqs. (3-4) are illustrated with respect to Eq. (1). The same methodology is applied to Eq. (2).

The displacement $\phi_{n}$ can be expanded as a sum of standing wave modes with slowly varying amplitudes. Taking into account the boundary conditions $\phi_{0}=\phi_{N+1}=0$, the standing wave modes are

$$
\mathrm{u}_{n}=\sin \left(\mathrm{n} q_{m}\right) ; q_{m}=\frac{m \pi}{N+1}, m=1 \ldots N
$$

The displacement of the $n^{\text {th }}$ pendulums is thus expressed as

$$
\phi_{n}=\underbrace{\sum_{m=1}^{N} A_{m} \sin \left(\mathrm{n} q_{m}\right) \exp \left(i \omega_{0} t\right)+c . c .}_{\phi_{0_{n}}}+\varepsilon \phi_{1_{n}}
$$

when it is deterministic and

$$
\tilde{\phi}_{n}=\underbrace{\sum_{m=1}^{N} \tilde{A}_{m} \sin \left(\mathrm{n} q_{m}\right) \exp \left(i \omega_{0} t\right)+\text { c.c. }}_{\tilde{\phi}_{0_{n}}}+\boldsymbol{\varepsilon} \tilde{\phi}_{1_{n}}
$$

if it contains uncertainty.

Substituting Eq. (6) into Eq. (4) leads to $N$ equations of the form

$$
\frac{\partial^{2} \phi_{1_{n}}}{\partial t^{2}}+\omega_{0}^{2} \phi_{1_{n}}=\sum_{\substack{m=1 \\ \text { +other terms }}}^{N}\left(m^{\text {th }} \text { secular tems }\right) e^{i \omega_{0} t}
$$

where the secular terms should be equated to zero to satisfy the condition of solvability of the multiple scales method. An equation similar to Eq. (8) in terms of $\tilde{\phi}_{n}$ is obtained when substituting Eq. (7) into Eq. (4).

Projecting the response on the standing-wave modes implies multiplying all terms by $\sin \left(n q_{m}\right)$ and summing over $n$ which leads to the generic complex equation of the $m^{\text {th }}$ amplitude $A_{m}$

$$
\begin{gathered}
2 i \omega_{0} \frac{\partial A_{m}}{\partial T}+i \omega_{0} c_{n} A_{m}+K_{n}\left(2 A_{m}-\cos \left[q_{m}\right] * G_{m}\right) \\
+S * \frac{2 K_{n}}{N+1} \sum_{n=1}^{N} \sin \left[\mathrm{n} q_{m}\right] \sum_{x=1}^{N} \cos \left[\mathrm{n} q_{x}\right] \sin \left[q_{x}\right]\left(A_{x}-\tilde{A}_{x}\right) \\
+\frac{3}{4} \alpha_{n} \sum_{j, k, l} A_{j} A_{k} A_{l}^{*} \Delta_{j k l, m}^{(1)}- \\
\frac{1}{(N+1)} F_{n} \exp (i \sigma T) \sum_{n=1}^{N} \sin \left[\mathrm{n} q_{m}\right]=0
\end{gathered}
$$

where $\Delta_{j k l, m}^{(1)}$ is the delta function defined in terms of the Kronecker deltas as demonstrated in $[2,3]$.

The introduced functions $G_{m}$ and $S$ are chosen according to the number and the positions of the stochastic pendulums in the chain, which is derived from the linear coupling. For purely deterministic case according to which the $n^{\text {th }}$ concerned pendulum is deterministic as well as its neighbors (case a, section 2.1), $G_{m}=2 A_{m}$ and $S=0$. If the deterministic pendulum is adjacent to a stochastic one, $G_{m}=A_{m}+\tilde{A}_{m}$ and $S=-1$ or $S=+1$ if the stochastic pendulum is, respectively, the previous (case b) or the next (case c). $\tilde{A}_{m}$ refers to the stochastic amplitude. When the concerned pendulum is stochastic (case d) and his neighbors are deterministic, $\tilde{A}_{m}$ 
replaces the deterministic amplitude $A_{m}$ in Eq. (9), $G_{m}=2 A_{m}$ and $S=0$.

The number of generated complex equations depends on the number and positions of stochastic pendulums. In fact, the $N$ equations obtained in a deterministic case becomes $N(p+$ $q+d)$ in a stochastic one, where $p$ is the number of stochastic pendulums, $q$ the number of deterministic pendulums neighbors of the stochastic ones and $d=1$ if the structure contains deterministic pendulums having deterministic neighbors and 0 otherwise.

To solve the obtained complex equations, a transformation of the amplitude to Cartesian form is needed

$$
A_{m}=\left(a_{m}+i b_{m}\right) \exp (i \sigma T)
$$

Substituting Eq. (10) into Eq. (9) and simplifying by $\exp (i \sigma T)$, one can obtain two generic equations for the real and imaginary parts of each amplitude $A_{m}$ of the form

$$
\begin{gathered}
\frac{\partial a_{m}}{\partial T}=\frac{\sigma}{2 \omega_{0}} b_{m}-\frac{c_{n}}{2} a_{m}-\frac{K_{n}}{2 \omega_{0}}\left(2 b_{m}-\cos \left[q_{m}\right] \operatorname{Img}\left(G_{m}\right)\right)-S * \\
\frac{1}{N+1} \frac{K_{n}}{\omega_{0}} \sum_{n=1}^{N} \sin \left[\mathrm{n} q_{m}\right] \sum_{x=1}^{N} \cos \left[\mathrm{n} q_{x}\right] \sin \left[q_{x}\right]\left(b_{x}-\tilde{b}_{x}\right)- \\
\frac{3}{8} \frac{\alpha_{n}}{\omega_{0}} \sum_{j, k, l}\left[a_{j} a_{k} b_{l}+b_{j} b_{k} b_{l}\right] \Delta_{j k l, m}^{(1)} \\
\frac{\partial b_{m}}{\partial T}=-\frac{\sigma}{2 \omega_{0}} a_{m}-\frac{c_{n}}{2} b_{m}+\frac{K_{n}}{2 \omega_{0}}\left(2 a_{m}-\cos \left[q_{m}\right] \operatorname{Real}\left(G_{m}\right)\right)+ \\
S * \frac{1}{N+1} \frac{K_{n}}{\omega_{0}} \sum_{n=1}^{N} \sin \left[\mathrm{n} q_{m}\right] \sum_{x=1}^{N} \cos \left[\mathrm{n} q_{x}\right] \sin \left[q_{x}\right]\left(a_{x}-\tilde{a}_{x}\right)+ \\
\frac{3}{8} \frac{\alpha_{n}}{\omega_{0}} \sum_{j, k, l}\left[a_{j} a_{k} a_{l}+a_{j} b_{k} b_{l}\right] \Delta_{j k l, m}^{(1)}-\frac{1}{2(N+1)} \frac{F_{n}}{\omega_{0}} \sum_{n=1}^{N} \sin \left[\mathrm{n} q_{m}\right]
\end{gathered}
$$

These obtained coupled algebraic equations are then numerically solved using the time integration Runge-Kutta method.

The stochastic analysis requires applying an uncertainty propagation method. In this paper, the statistical LHS method [16] is used. The choice of this method is due to its simple implementation consisting on a generation of a succession of deterministic computations according to a set of random variables and its reduced computing time with respect to the very time-consuming $\mathrm{MC}$ method. In fact, a great number of samples are typically required in $\mathrm{MC}$ to achieve good accuracy while partitioning the variability space into regions of equal probability and picking up one sampling point in each region allow reducing the number of samples needed for the LHS method implementation.

\section{NUMERICAL SIMULATIONS}

The uncertainty propagation using the LHS method through the generic discrete model proposed in this paper is numerically implemented in order to illustrate the effects of uncertainties on the behavior of a structure composed of three coupled pendulums $(N=3)$ for which, the design parameters are listed in Tab. 1.

TABLE 1. DESIGN PARAMETERS OF THE THREE COUPLED PENDULUMS STRUCTURE.

\begin{tabular}{cccccc}
\hline $\begin{array}{c}m \\
(\mathrm{~kg})\end{array}$ & $\begin{array}{c}l \\
(\mathrm{~m})\end{array}$ & $\begin{array}{c}k \\
\left(\mathrm{~N} . \mathrm{m}^{-1}\right)\end{array}$ & $\begin{array}{c}\alpha \\
\left(\mathrm{kg} . \mathrm{m}^{2} . \mathrm{s}^{-1}\right)\end{array}$ & $\begin{array}{c}f \\
(\mathrm{~N})\end{array}$ & $\begin{array}{c}\omega_{0} \\
\left(\mathrm{rad} . \mathrm{s}^{-1}\right)\end{array}$ \\
\hline 0.25 & 0.062 & $9.10^{-4}$ & 0.01 & 0.01 & 12.58 \\
\hline
\end{tabular}

The length of the first pendulum is supposed to be uncertain and varies such as

$$
l_{s}=l\left(1+\delta_{l} \xi_{l}\right)
$$

where $\delta_{l}$ and $\xi_{l}$ are respectively chosen dispersion level and Gaussian random variable.

The problem is numerically time-consuming when using the time integration Runge-Kutta method, in each LHS samples, to solve the obtained algebraic equations due to the necessity to sufficiently refine frequency step and vary initial conditions to obtain more stable solutions. This constraint imposes minimizing as possible the number of samples for the LHS method. 200 samples are therefore generated.

A statistical analysis of the frequency responses dispersion, both in generalized (modal) and physical coordinates, is carried out in order to investigate the robustness of the multimode branches against uncertainties in the multistability domain. Indeed, the results are presented at first in term of envelope of the displacement amplitude of each pendulum (Figs. 3-5). Then, a comparative study with respect to the deterministic solutions, presented by Fig. 2, permits to show the effect of uncertainties in the multistability domain through the quantification of the amplitude and frequency ranges variation (Tab. 2 and Fig. 7).

Deterministic frequency responses in modal and physical coordinates are shown in Fig. 2. In the multistability domain, different stable solutions exist and are generated by the modal interactions between the responses which are driven by the collective dynamics. Six stable solutions could thus be obtained in the multistability domain. The obtained stable branches can be classified as Single (SM), Double (DM) and Triple mode (TM) solutions. In fact, the only SM branch corresponds to the null trivial solution of the second amplitude and is presented by black curve in Fig. 2 (A). The DM branches, which are presented by red curves, correspond to the solutions for the first and third amplitudes, whereas the TM solutions (blue curves) result from the coupling between the three pendulums. A correspondence in term of bifurcation points between the amplitudes with respect to each branch type is observed. It is generated by the bifurcation topology transfer. 
(A)
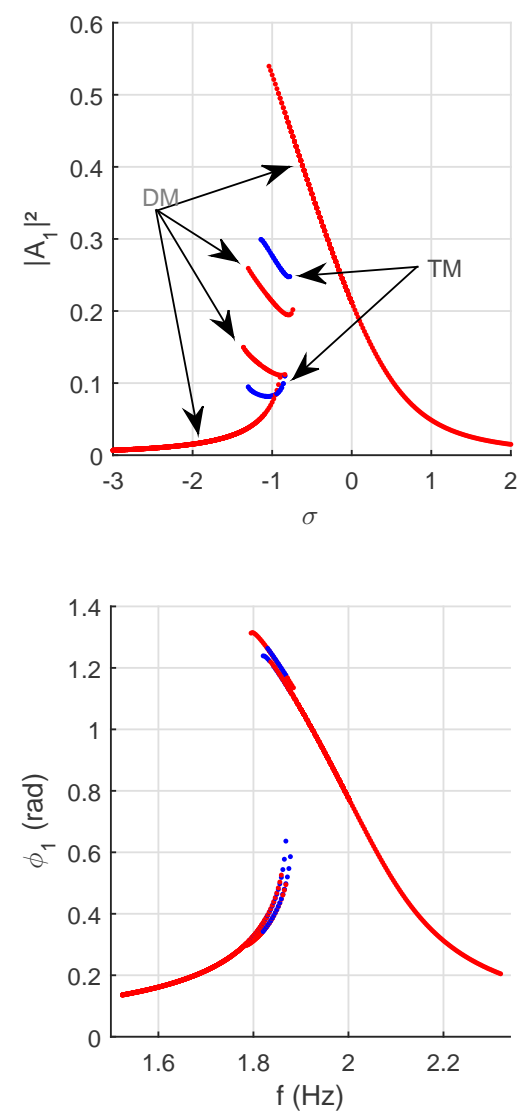

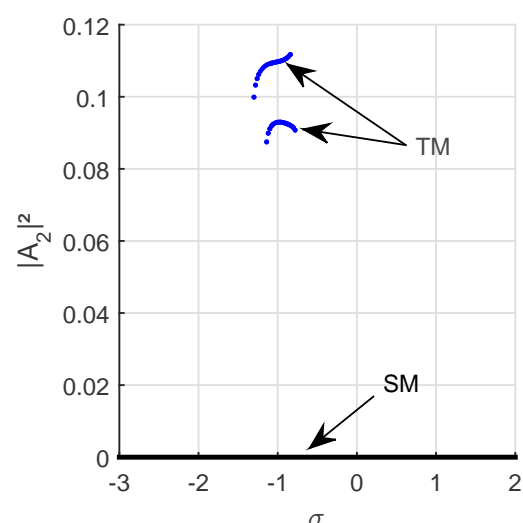

(B)

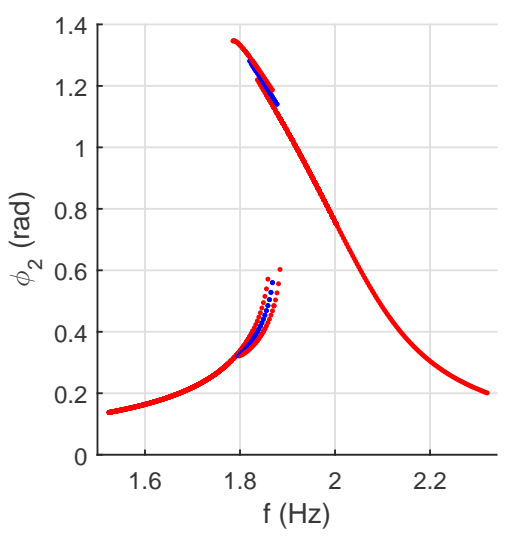

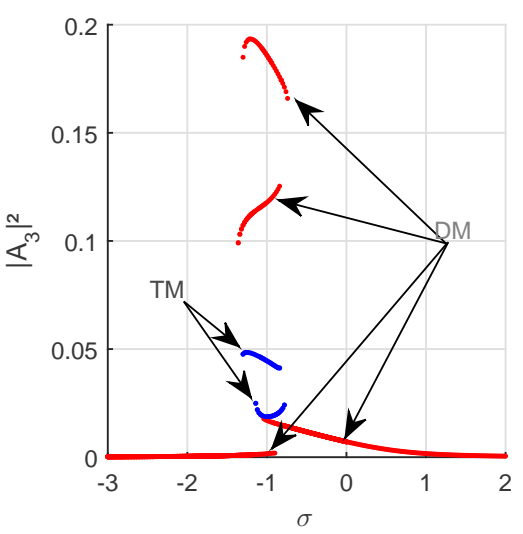

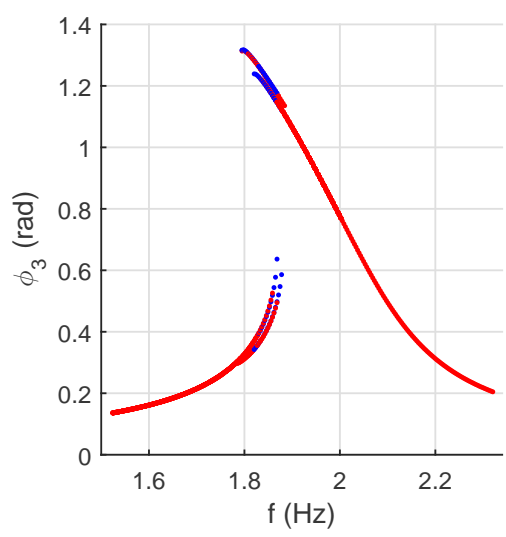

FIGURE 2. DETERMINISTIC RESPONSE AMPLITUDES OF THE PENDULUMS IN (A) GENERALIZED AND (B) PHYSICAL COORDINATES.

In order to enable comparative study of the obtained results with respect to the deterministic ones, the samples of the LHS method are generated according to the initial conditions fixed for the deterministic case. The shown discontinuity in the envelope curves is due to the "lack" of initial conditions. However, it is important to note that varying more the initial conditions makes computation prohibitive and it still difficult to cover all possible solutions and so obtain smooth curves.

To illustrate the stochastic effect on the structure behavior when uncertainty is taken into account, two dispersion levels are considered: $2 \%$ and $5 \%$. For $\delta_{l}=2 \%$, the envelopes of the response amplitudes in modal coordinates are shown in Fig. 3, while Fig. 4 illustrates the amplitude envelopes for $\delta_{l}=5 \%$. Moving to physical coordinates, response envelopes are presented by Fig. 5 for different dispersion levels.

It is clearly shown that increasing the dispersion level affects more the variability of the response amplitudes, both in modal and physical coordinates. Large variability is obtained for $\delta_{l}=5 \%$ and the overlap of envelopes makes detecting the extreme statistics of adjacent branches more difficult.

The effects of uncertainties on the dispersion of the response amplitudes depend on the position of each concerned pendulum with respect to the stochastic one. In fact, as shown in Figs. 3-4, the envelope width differs when moving from one pendulum to another. The greatest impact is on the response of the first pendulum since it contains the uncertain parameter. The second one is less affected while the behavior of the third pendulum remains deterministic.

When increasing the dispersion level, all branches are larger in terms of amplitude and frequency ranges. Consequently, the multimode solutions are enhanced and the multistability domain is wider.

In addition to the curves presented in Fig. 5 showing the response envelopes in physical coordinates, Tab. 2 and Fig. 7 illustrate the effects of uncertainties through calculating the intervals of variation of the amplitude $(\Delta \phi)$ and frequency $(\Delta f)$ ranges of the multistability domain, between extreme bifurcation points, according to the example showed in Fig. 6. As mentioned above and proved through Fig. 7, the first pendulum response is more affected by uncertainties than the second since its length is stochastic. The third pendulum has deterministic behavior because no coupling terms relate its equation with the one associated to the stochastic pendulum. 
More important variation is detected for the frequency ranges $(\Delta f)$ than the amplitude ones $(\Delta \phi)$. When computing the ratio $\Delta f / \Delta \phi$, one can show the impact of uncertainties on the nonlinearity of the structure. Indeed, dispersion and nonlinearity are proportional. Consequently, the nonlinear aspect of the structure is enhanced.

(A)
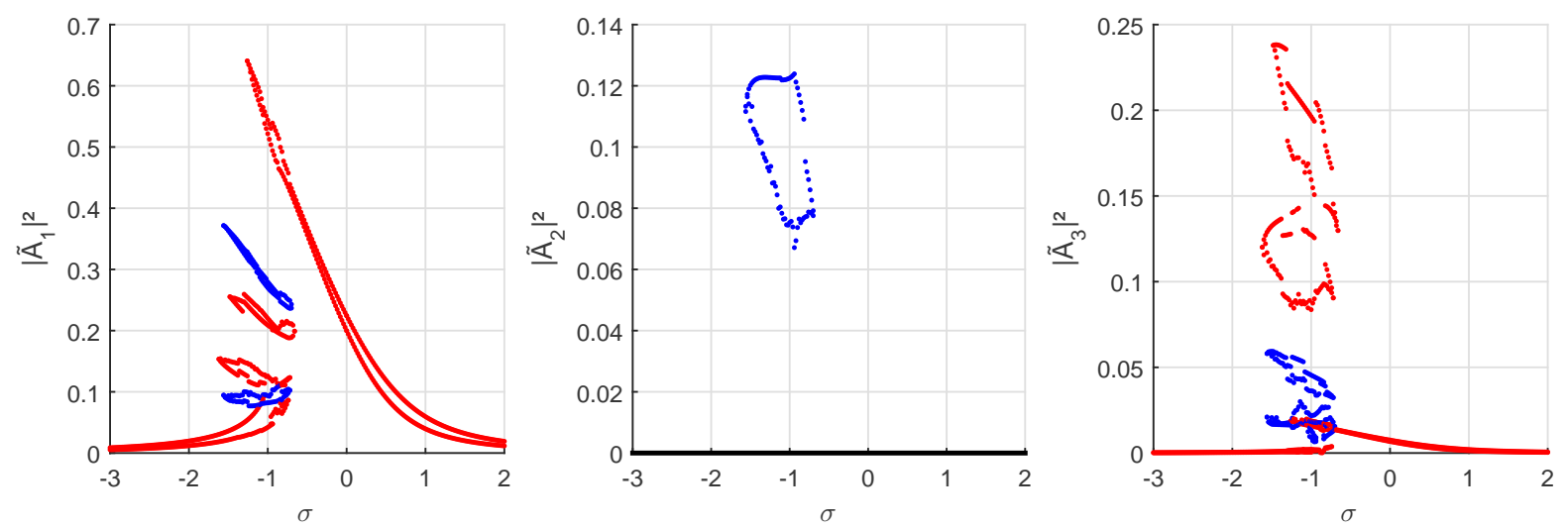

(B)
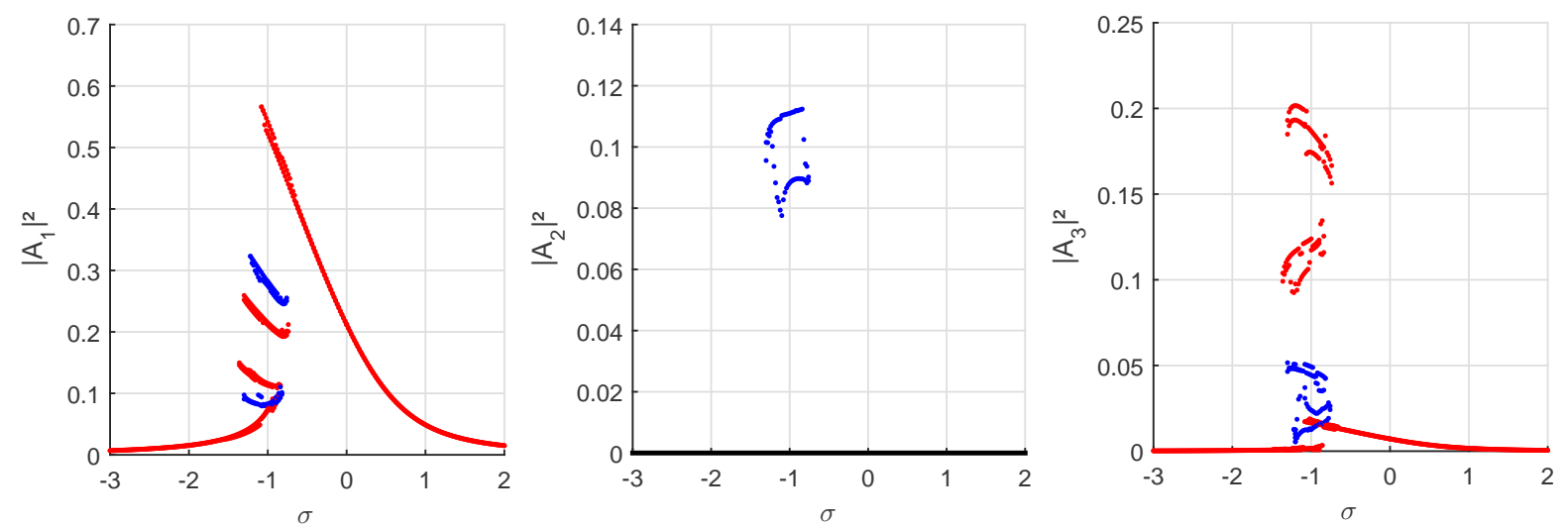

(C)
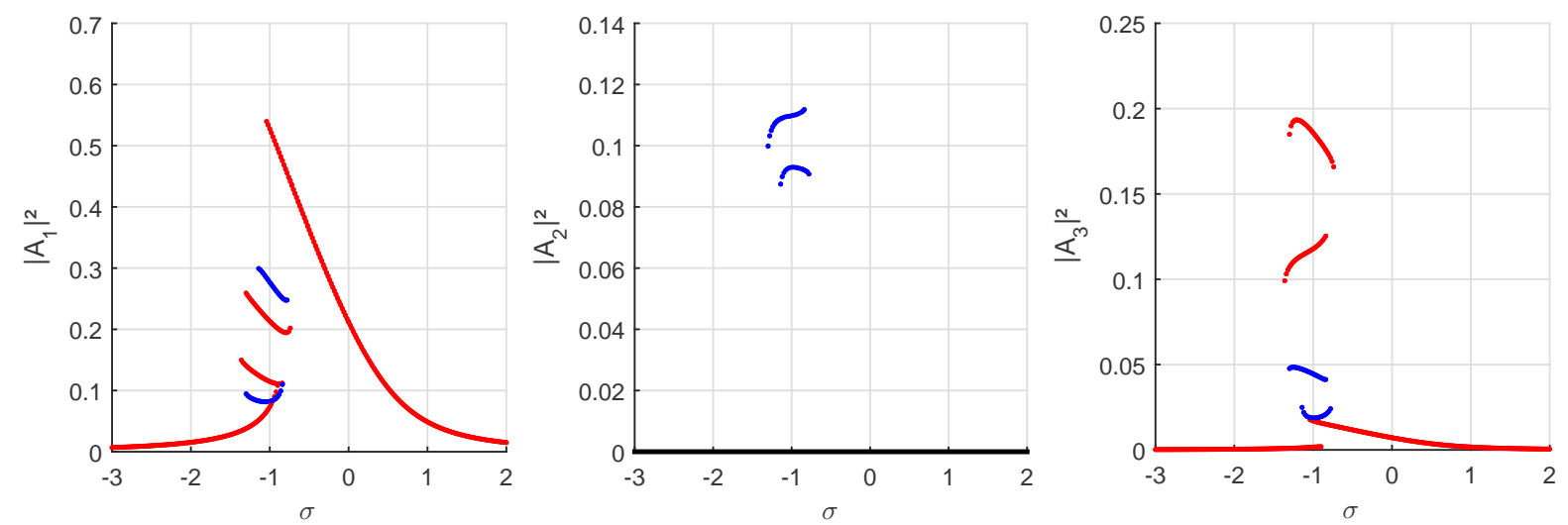

FIGURE 3. ENVELOPES OF RESPONSE AMPLITUDES OF THE (A) FIRST, (B) SECOND AND (C) THIRD PENDULUM IN GENERALIZED COORDINATES FOR $\delta_{l}=2 \%$. 
(A)
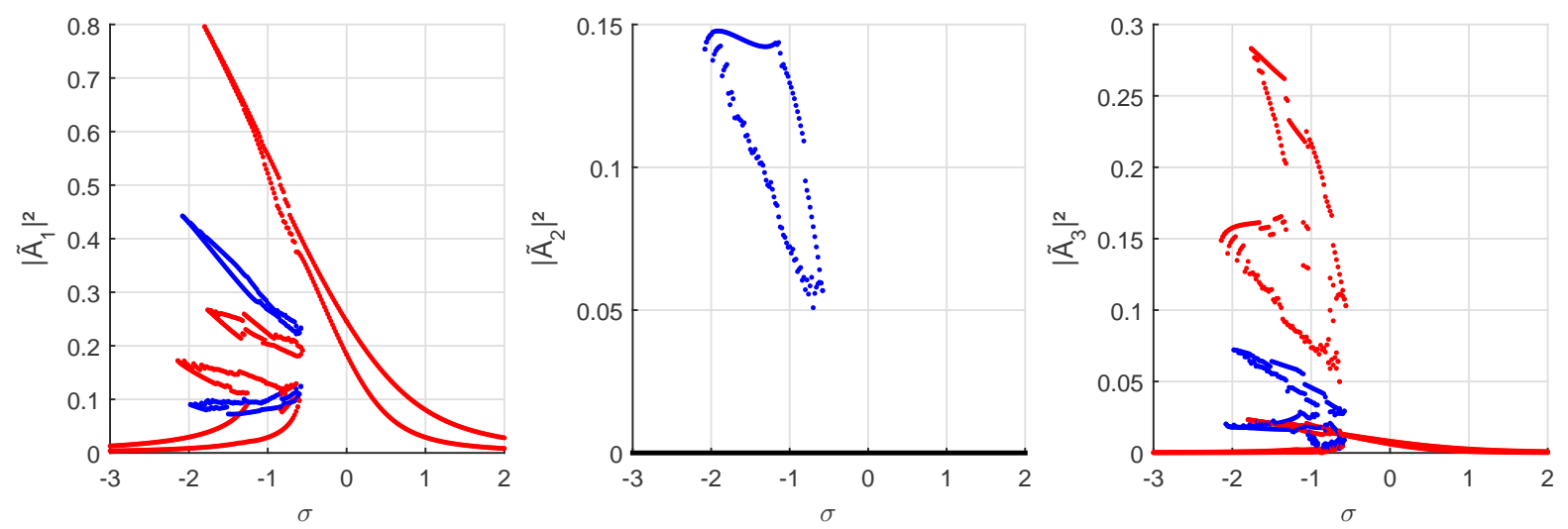

(B)
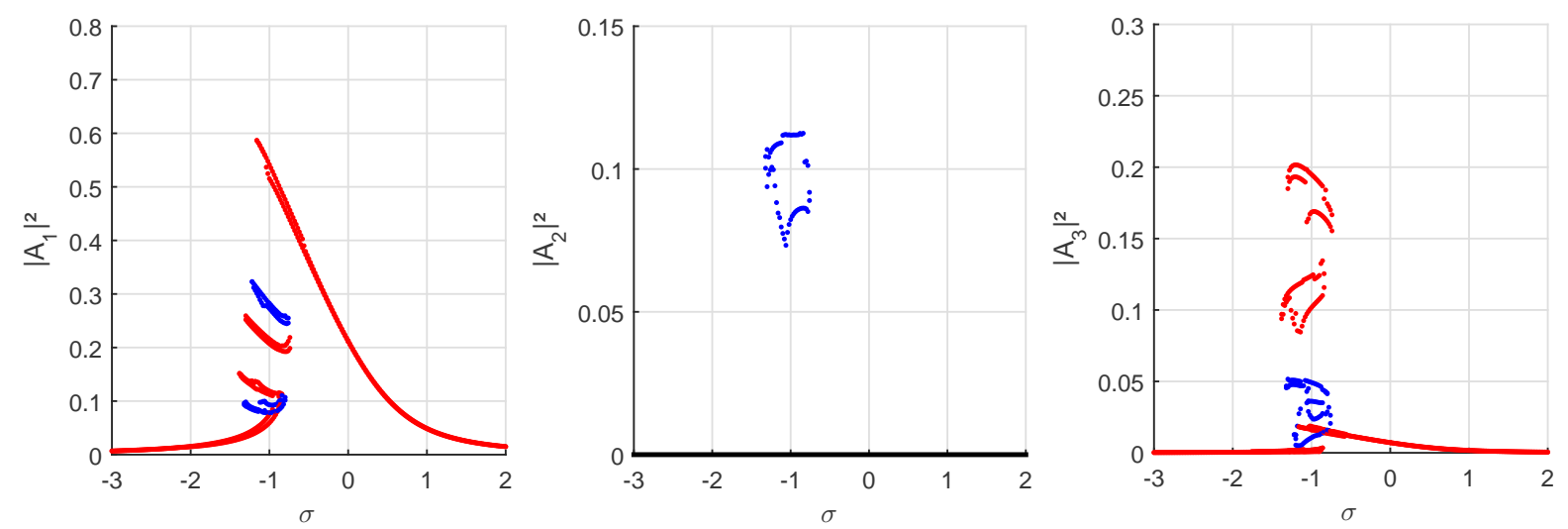

(C)
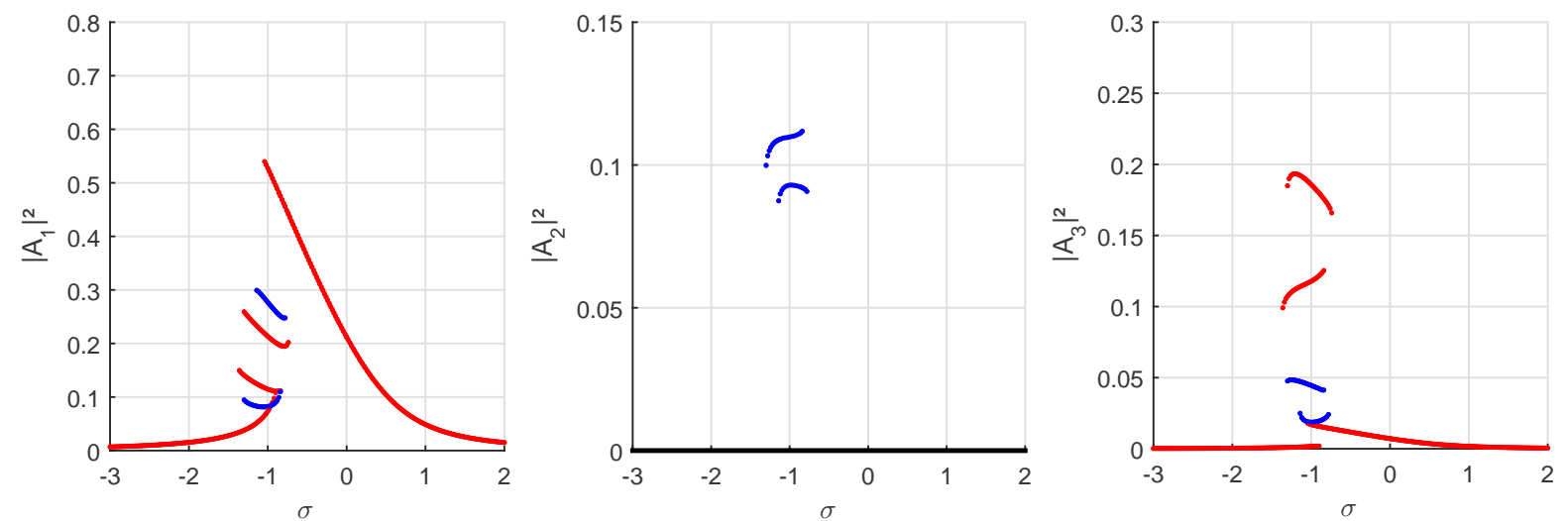

FIGURE 4. ENVELOPES OF RESPONSE AMPLITUDES OF THE (A) FIRST, (B) SECOND AND (C) THIRD PENDULUM IN GENERALIZED COORDINATES FOR $\delta_{l}=5 \%$. 
(A)
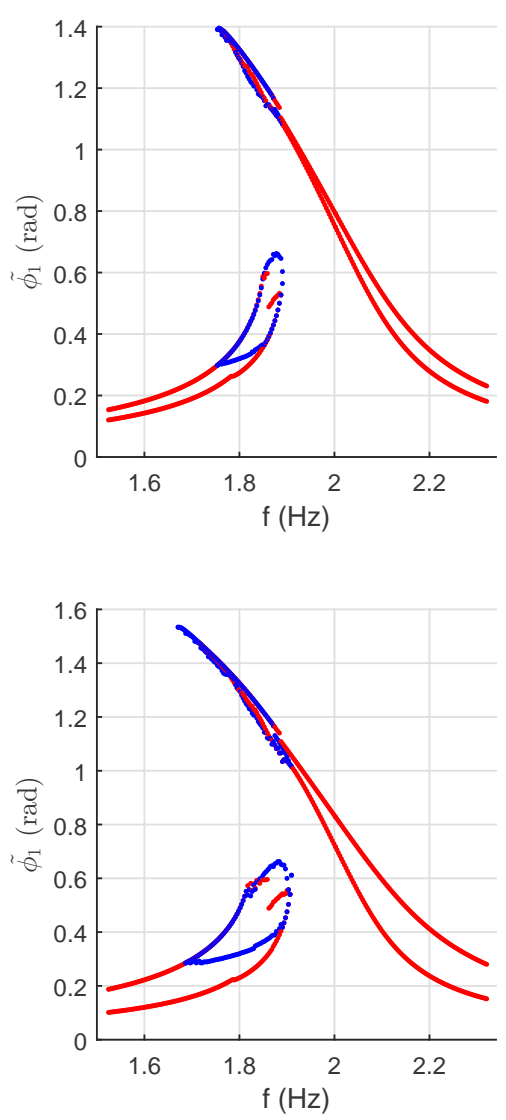

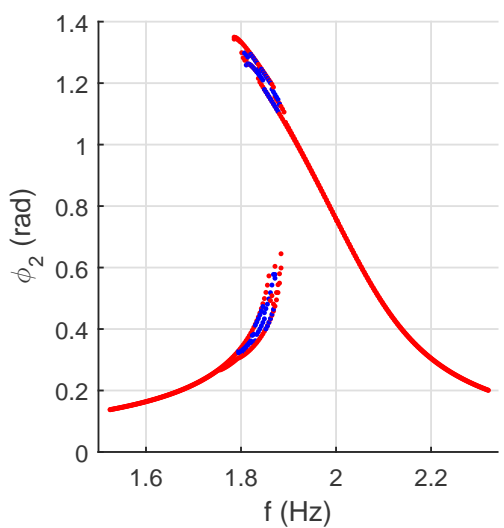

(B)

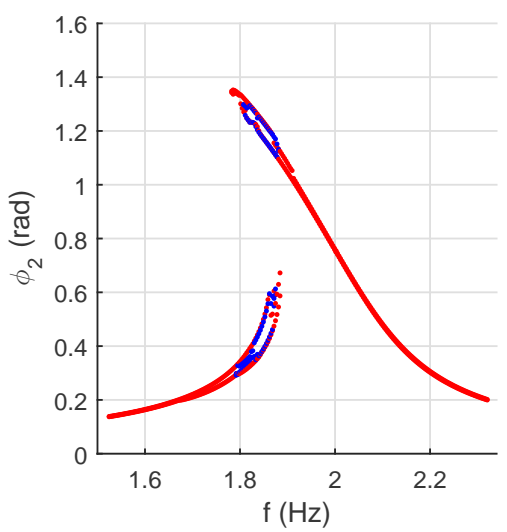

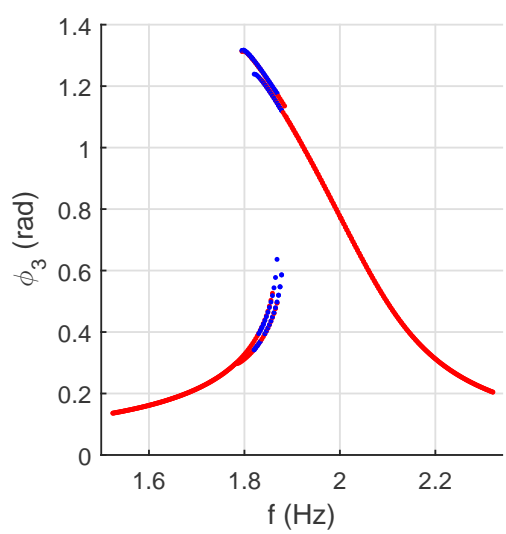

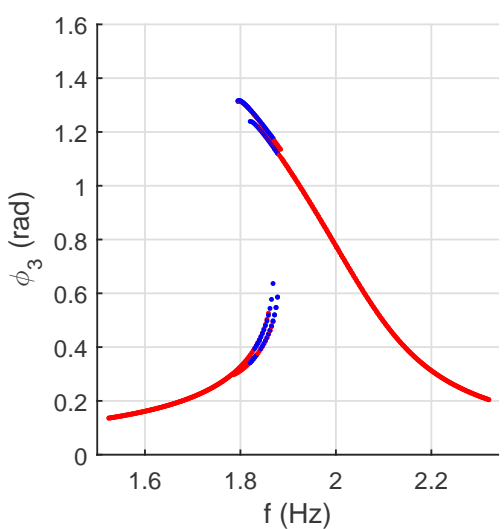

FIGURE 5. ENVELOPES OF RESPONSE AMPLITUDES OF THE PENDULUMS IN PHYSICAL COORDINATES FOR (A) $\delta_{l}=2 \%$ AND (B) $\delta_{l}=5 \%$.

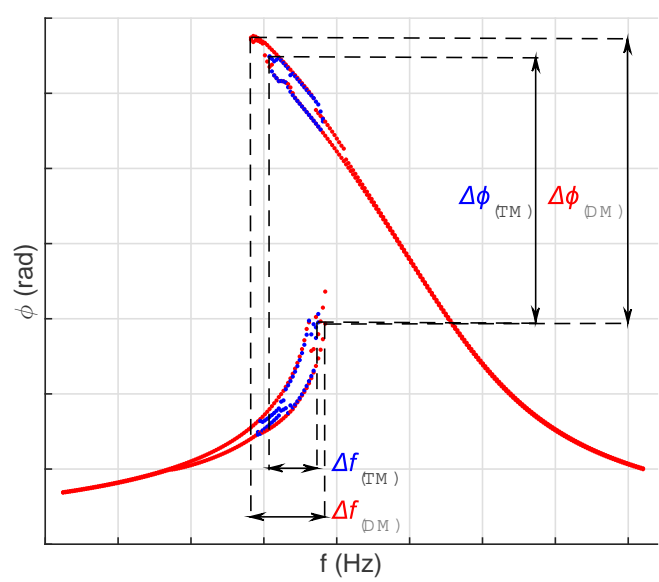

FIGURE 6. EXAMPLE OF MEASURED AMPLITUDE AND FREQUENCY RANGES OF THE MULTISTABILITY DOMAIN ACCORDING TO THE DM AND TM.
TABLE 2. STOCHASTIC AMPLITUDE AND FREQUENCY RANGES OF THE MULTISTABILITY DOMAIN, ACCORDING TO THE DM AND TM, WITH RESPECT TO THE DETERMINISTIC RANGES.

\begin{tabular}{cccccc}
\hline & & \multicolumn{2}{c}{$\mathrm{DM}$} & \multicolumn{2}{c}{$\mathrm{TM}$} \\
& dof & $\delta_{l}=2 \%$ & $\delta_{l}=5 \%$ & $\delta_{l}=2 \%$ & $\delta_{l}=5 \%$ \\
\hline \multirow{4}{*}{$\Delta f(\%)$} & 1 & 172.86 & 272.86 & 165.06 & 287.95 \\
& 2 & 110.47 & 113.95 & 143.18 & 165.91 \\
& 3 & 100 & 100 & 100 & 100 \\
\hline \multirow{3}{*}{$\Delta \phi(\%)$} & 1 & 104.76 & 114.15 & 113.29 & 126.44 \\
& 2 & 100.94 & 103.10 & 100.83 & 97.39 \\
& 3 & 100 & 100 & 100 & 100 \\
\hline \multirow{2}{*}{$\Delta \phi$} & 1 & 165.88 & 240.00 & 145.61 & 227.19 \\
& 2 & 109.48 & 110.34 & 140.98 & 168.85 \\
& 3 & 100 & 100 & 100 & 100 \\
\hline
\end{tabular}



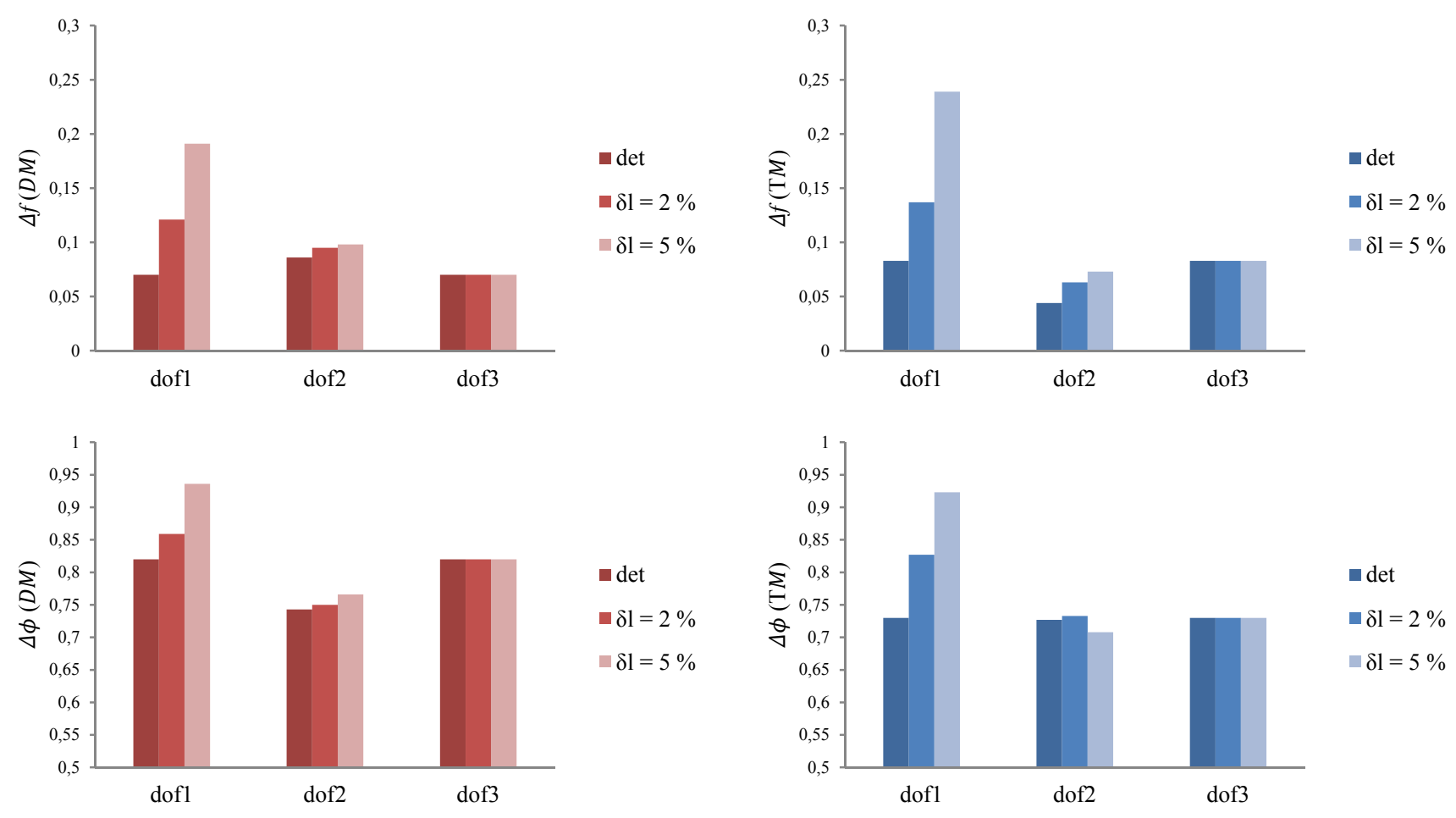
FIGURE 7. EVOLUTION OF THE FREQUENCY AND AMPLITUDE RANGES OF THE MULTISTABILITY DOMAIN
WITH RESPECT TO DETERMINISTIC ONES ACCORDING TO THE DM AND TM.

\section{CONCLUSION}

Under the hypothesis of small imperfections, a generic discrete analytical model combining the multiple scales method, the perturbation theory and a standing-wave decomposition is proposed in order to investigate the collective dynamics of a nonlinear periodic structure composed of three linearly coupled pendulums under parametric uncertainties. The statistical LHS method permits to propagate uncertainty through the proposed model.

The analysis of the dispersion, in terms of variability of the frequency and amplitude intervals of the multistability domain according to different dispersion levels of uncertain input parameters, shows the effects of uncertainties on the stability and nonlinearity of the structure. The obtained results prove that the presence of uncertainties in a periodic structure enhances the multimode solutions introduced by the collective dynamics and interactions between pendulums by obtaining additional stable branches and widening the multistability domain. The uncertainties enhance also the nonlinear aspect of the structure. Further works concern the extension of the proposed method to large-size periodic structures.

\section{REFERENCES}

[1]. Boechler N., Daraio C., Narisetti R. K., Ruzzene M., Leamy M.J., 2010, "Analytical and experimental analysis of bandgaps in nonlinear one dimensional periodic structures", In Tsung-Tsong $\mathrm{Wu}$ and ChienChing Ma, editors, IUTAM Symposium on Recent Advances of Acoustic Waves in Solids, Vol 26 of IUTAM Bookseries, pp. 209-219. Springer Netherlands.

[2]. Lifshitz R., Cross M. C., 2003, "Response of parametrically driven nonlinear coupled oscillators with application to micromechanical and nanomechanical resonator arrays", Physical Review B, 67, 134302.

[3]. Bitar D., Kacem N., Bouhaddi N., Collet M., 2015, "Collective dynamics of periodic nonlinear oscillators under simultaneous parametric and external excitations", Nonlinear Dynamics, 82, pp. 749-766.

[4]. Gutschmidt S., Gottlieb O., 2012, "Nonlinear dynamic behavior of a microbeam array subject to parametric actuation at low, medium and large dc-voltages", Nonlinear Dynamics, 67(1), pp. 1-36.

[5]. Manktelow K., Leamy M. J., Ruzzene M., 2014, "Weakly nonlinear wave interactions in multi-degree of freedom periodic structures", Wave Motion 51, pp. 886904.

[6]. Andreassen E., Manktelow K., Ruzzene M., 2015, "Directional bending wave propagation in periodically perforated plates", Journal of Sound and Vibration, 335, pp. 187-203.

[7]. Kissel G. J., 1988, "Localization in disordered periodic structures", Ph.D. thesis, Massachusetts Institute of Technology. 
[8]. Cha P. D., Pierre C., 1997, “A statistical investigation of the forced response of finitel nearly periodic assemblies", Journal of Sound and Vibration, 203(1), pp. 158-168.

[9]. Koch R. M., 2003, "Structural Dynamics of Large Space Structures Having Random Parametric Uncertainties", International Journal of Acoustics and Vibration, 8(2), pp. 95-103.

[10]. Jallouli A., Kacem N., Bouhaddi N., 2015, "Nonlinear dynamics of a 2D array of coupled pendulums under parametric excitation", In 5th ECCOMAS Thematic Conference on Computational Methods in Structural Dynamics and Earthquake Engineering (COMPDYN 2015), pp. 8.

[11]. Tjavaras A. A., Triantafyllou M. S., 1996, "Non-linear response of two disordered pendula", Journal of Sound and Vibration, 190(1), pp. 65-76.
[12]. Hai-Quing X., Yi T., 2006, "Parametrically driven solitons in a chain of nonlinear coupled pendula with an impurity", Chinese Physics Letters, 23(6), pp. 15-44.

[13]. Chen W., Hu B., Zhang H., 2002, "Interactions between impurities and nonlinear waves in a driven nonlinear pendulum chain", Physical Review Letters, 64, 134302.

[14]. Zhu Y., Chen W., Lu L., 2003, "Experiments on the interactions between impurities and solitary waves in lattice model", Science in China (Series G), 46(5).

[15]. Bitar D., Kacem N., Bouhaddi N., 2015, "Multi-mode solutions in a periodic array of coupled nonlinear pendulums under primary resonance", 11th International Conference on Engineering Vibration Ljubljana, Slovenia, September 7-10.

[16]. Helton J.C., Davis F.J., 2003, "Latin hypercube sampling and the propagation of uncertainty in analyses of complex systems", Reliability Engineering \& System Safety, 81(1), pp. 23-69. 